Rev. Elev, Méd. vét. Pays trop., 1972, 25 (3) : 375-382

\title{
Le polymorphisme biochimique chez les bovins : Etude de la Glucose-6-Phosphate Déshydrogénase
}

\author{
par J.-P. PETIT $\left(^{*}\right)$ et R. QUEVAL $\left({ }^{* *}\right)$
}

\begin{abstract}
RESUME
Après avoir rappelé le rôle de la glucose-6-phosphate déshydrogénase dans le cycle des pentoses chez les animaux, les auteurs déterminent la déficience en cet enzyme érythrocytaire chez 108 zébus arabes et chez 61 taurins Kouri. L'analyse statistique complète des résultats, reprenant les données brutes de NAIK et BAXI sur 6 autres races de zébus Indien et Pakistanais, ne permet pas de retenir comme différence biologique entre zébus et taurins la déficience en G-6-PD. On ne peut pas non plus établir de corrélation avec la classification des zébus Indiens de JOSHI et PHILLIPS. On peut actuellement penser qu'il s'agit seulement d'une caractéristique raciale chez les bovins.
\end{abstract}

\section{INTRODUCTION}

Les propriétés zootechniques et biologiques des bovins ne suffisent plus à la connaissance approfondie des races.

Il devient impératif de trouver de nouveaux critères quantitatifs ou même qualitatifs correspondant génétiquement aux propriétés biologiques que l'homme cherche à exploiter. Dans cette voie se trouve l'approfondissement des définitions raciales par détermination de la nature des hémoglobines, des groupes sanguins, des composants du plasma, etc., tous critères biochimiques.

La glucose-6-phosphate déshydrogénase, par son importance dans le métabolisme, pourrait avoir un intérêt de ce point de vue. Son étude s'intègre dans celle des systèmes polymorphiques chez les espèces animales domestiques, particulièrement développés chez les bovins, où trente-trois systèmes immunogénétiques et chémogénétiques ont été analysés.

(*) I.E.M.V.T., Laboratoire de Biochimie, 10, rue Pierre Curie, 94 Maisons-Alfort, France.

(**) I.E.M.V.T., Laboratoire de Recherches Vétérinaires de Farcha, B.P. no 433, Fort-Lamy, Tchad.

\section{LA GLUCOSE-6-PHOSPHATE DESHYDROGENASE CHEZ L'HOMME ET CHEZ L'ANIMAL}

Le rôle de la glucose-6-phosphate déshydrogénase (G-6-PD, NADD ou oxydoréductase) a été montré en 1931 par WARBURG (13). Elle a été isolée à partir de levures dès 1950 par KORNBERG (8), puis à partir d'hématies en 1960 par MARKS et collab. (9). Cet enzyme permet l'oxydation du glucose-6phosphate en 6-phospho-gluconolactone au début du cycle des pentoses (fig. 1); le coferment étant ici le nicotinamide-adéninedinucléotide (NADP) et non le NAD (***). Ce cycle amène à l'acide ribo-nucléique (ARN) par l'intermédiaire du ribulose-6-phosphate et des nucléosides.

Le cycle des pentoses a une importance particulière chez les mammifères dans les tissus mammaires pendant la lactation et, en général, dans tous les tissus des animaux supérieurs en voie de prolifération (embryons, glandes).

$(* * *)$ Voir liste des abréviations. 


\title{
Glucose_ 6 _ phosphate
}

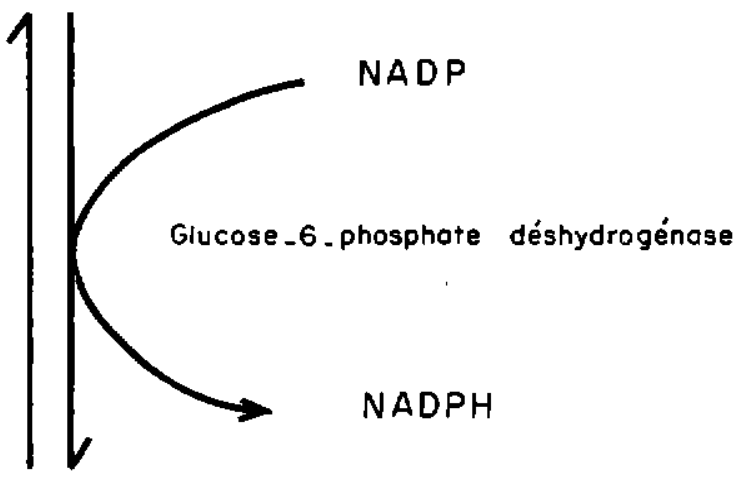

\section{6. phosphogluconolactone}

\begin{abstract}
Fig. 1. - Oxydation de glucose-6-phosphate au début du cycle des pentoses, cette réaction étant catalysée par la G-6-PD et couplée à l'hydrogénation du nucléotide-adénine-dinucléotide phosphorylé (NADP). Ce cycle permet la synthèse de l'ARN.
\end{abstract}

On trouve cet enzyme surtout dans les hématies, dans le foie, les reins et la moelle osseuse. Il augmente considérablement dans les cas de carcinomes hépatiques; lors du métabolisme hépatique normal, la moitié du glucose est utilisée par la voie des hexoses monophosphates et des pentoses, l'autre moitié prenant la voie d'Embden-Meyerhoff $\left({ }^{*}\right)$.

\section{Chez l'homme}

CARSON et collab. (1956) (4) ont montré que le déficit en glucose-6-phosphate déshydrogénase des hématies est la cause d'accidents hémolytiques après absorption de certains antipaludéens de synthèse tels que la primaquine (primaquine-sensibilité), après ingestion de substances chimiques telles que les nitrofuranes, la phénacétine, les sulfonamides, la naphtaline ou de légumes comme la fève (Vicia fava).

La sensibilité des hématies à la primaquine, due à un déficit enzymatique en G-6-PD, est liée à une prédisposition raciale; ainsi dans la race noire américaine, ce déficit enzymatique a été observé chez environ 13 p. 100 des hommes et 3 p. 100 des femmes. Dans les régions périméditerranéennes, les pourcentages sont sensiblement les mêmes parmi diverses races ou ethnies telles que les sardes, les grecs, les juifs, etc.

(*) Voir liste des abréviations.
Le polymorphisme génétique de la G-6-PD érythrocytaire chez l'homme a été étudié par BOYER, PORTER et WEILBACHER (1962) (3), KIRKMAN et HENDRICKSON (1963) (7) qui ont établi l'existence de deux variétés normales de G-6-PD qui diffèrent par leur mobilité électrophorétique. Le type $\mathbf{B}$ est le plus répandu et le type $A$ ne se rencontre que chez les sujets de race noire (phénotypes normaux $\mathrm{A}^{+}$et $\mathrm{B}^{+}$). Deux autres variétés normales : $\mathrm{C}^{+}$(ou Baltimore) et Ibadan paraissent plus rares que les types $\mathrm{A}^{+}$et $\mathrm{B}^{+}$.

Le déficit en G-6-PD des hématies peut avoir pour origine : l'absence d'enzyme ou bien la substitution à l'enzyme normale active d'un variant moins actif ou inactif. Fréquent chez les Noirs, le déficit en G-6-PD ne frappe que les sujets de type $A$. Le Blanc déficient est toujours du type B (phénotypes déficients respectivement $\mathrm{A}^{-}$et $\mathrm{B}^{-}$). La transmission du type de G-6-PD est héréditaire.

\section{Chez les animaux}

La G-6-PD érythrocytaire a été mise en évidence par BAXI et collab. (1963) (1) chez les équidés (cheval, mulet, âne), le chien, le porc et le lapin.

NAIK, BAXI et BHATIA (1963) (12) rapportent la présence de l'enzyme érythrocytaire chez 60 p. 100 des bovins importés, de race Jersey. 
KHANOLKAR et collab. (1963) (6) notent l'absence totale de la G-6-PD chez le mouton et la chèvre.

Plus récemment, NAIK et BAXI (1968) (11) l'ont étudiée parmi six races bovines des Indes et indiquent les fréquences raciales de l'enzymopénie: Malvi (29,5 p. 100), Gir (31,0 p. 100), Rath (31,75 p. 100), Khillari (35,72 p. 100), Dangi (39,55 p. 100) et Kankrej (50,0 p. 100).

En résumé, selon cet ensemble de travaux, on note 3 grands groupes parmi les animaux domestiques :

- groupe I, présence constante de la G-6-PD chez tous les individus, c'est le cas du cheval et du lapin,

- groupe II, espèces telles que le bœuf, le chien et le porc possédant soit des individus normaux, soit des individus déficitaires en G-6-PD,

- groupe III : les petits ruminants tels que le mouton et la chèvre se caractérisant par une absence totale de l'enzyme érythrocytaire.

Ce travail, après détermination de l'activité de la G-6-PD chez les 108 zébus et 61 taurins, reprend l'analyse statistique faite par NAIK et BAXI en 1968, pour essayer de tirer des conclusions élargies tenant compte des nouvelles déterminations.

\section{MATERIEL ET METHODE}

La détermination de l'activité de la G-6-PD a porté sur un groupe de cent huit zébus arabes (Bos indicus) et un lot de soixante et un taurins Kouris (Bos taurus), tous de sexe mâle.

Les échantillons de sang ont été prélevés et recueillis aseptiquement sur héparine, puis examinés immédiatement ou conservés environ 18 heures à une température de $+4^{\circ} \mathrm{C}$.

Ce mode opératoire est impératif, si l'on ne veut pas s'exposer à trouver des activités nulles pour des animaux en réalité à activité enzymatique normale.

Une variante du test au bleu crésyl brillant de MOTULSKY et CAMPBELL-KRAUT (1961) (10) a été utilisée pour la détermination semi-quantitative de cet enzyme érythrocytaire.

\section{a) Principe}

Le test est basé sur le principe suivant: le NADPH (nicotinamide-adénine-dinucléotide phosphorylé, dérivé hydrogène) (*) formé grâce à l'action de la G-6-PD réduit le bleu crésyl brillant en un composé incolore.

Le bleu crésyl brillant remplit deux fonctions dans ce test : d'une part, il est l'indicateur de la formation du NADPH et, d'autre part, il stimule l'activité du cycle des pentoses.

\section{b) Mode opératoire}

La technique suivie a été mise au point par BERGER (1965) (2).

A partir des échantillons sanguins héparinés, on prélève à la micropipette $0,05 \mathrm{ml}$ de sang total que l'on expulse dans un tube à hémolyse contenant 2,5 ml d'eau distillée. Après une agitation vigoureuse, on laisse $10 \mathrm{mn}$ pour obtenir une hémolyse complète. Dans les tubes réactifs, un $\mathrm{ml}$ d'hémolysat est additionné à $0,5 \mathrm{ml}$ d'un substrat tamponné à $\mathrm{pH} 8,5$ [tris (hydroxyméthyl) aminométhane]. Une huile minérale est ensuite ajoutée $(2 \mathrm{ml})$ pour prévenir l'oxydo-réduction du colorant. Les tubes sont placés au bain marie à $37^{\circ} \mathrm{C}$ et à l'obscurité (temps zéro).

Quarante cinq mn après la mise en route du test, les temps de décoloration sont notés toutes les $5-10 \mathrm{mn}$.

Avec un sang normal, la décoloration totale apparaît entre 50 et $100 \mathrm{mn}$. Les échantillons encore colorés après 2 heures sont considérés comme déficitaires en G-6-PD.

\section{c) Analyses statistiques}

Elles ont été menées sur ordinateur pour saisir toutes les interprétations possibles et pour garantir la précision des résultats.

La stratégie suivie se résume ainsi : test $\mathrm{X}^{2}$ globaux, puis partiels pour localiser les différences significatives au seuil habituel de $5 \mathrm{p}$. 100. Des particularités apparaissant ainsi pour les races Kankrej et Dangi, les calculs ont été repris pour observer l'influence de chacune de ces races sur les résultats antérieurs et pour

${ }^{*}$ ) Voir liste des abréviations. 
déterminer une éventuelle communauté entre elles.

\section{RESULTATS}

Séparés par groupe de bovins et par race, ils sont rassemblés dans le tableau $\mathrm{I}$, et com- parés globalement dans le tableau II. On constate immédiatement que tous les $\mathrm{X}^{2}$ globaux incluant la race Kankrej sont significatifs alors que la seule exclusion de cette race ne permet plus de dire que les variations observées dans la déficience en G-6-PD soient dues à un autre facteur que le hasard.

TABLEAU $\mathrm{N}^{\circ} \mathrm{I}$

Dêtermination de la Glucose-b-Phosphate deshydrogënase chez huit races de bovins et pourcentage des animaux trouvës déficients dans chacune d'e11es.

\begin{tabular}{|c|c|c|c|c|c|c|}
\hline N a t u r e d & $b \circ v i n s$ & \multirow{2}{*}{$\begin{array}{l}\text { Nombre } \\
\text { total }\end{array}$} & \multicolumn{2}{|c|}{$\begin{array}{c}\text { Données brutes : nb. } \\
\text { d'animaux ayant une } \\
\text { G } 6-\text { PD }\end{array}$} & \multirow{2}{*}{$A u t e u r s$} & \multirow{2}{*}{$\begin{array}{l}\text { Calculs } \\
\text { pourcentage } \\
\text { déficience } \\
\text { recalculés }\end{array}$} \\
\hline Groupe & $\mathrm{R}$ a c e & & $\begin{array}{c}\text { normale } \\
+\end{array}$ & $\begin{array}{c}\text { déficiente } \\
-\end{array}$ & & \\
\hline Bos taurus & (1) Kouri & 61 & 39 & 22 & Petit (J.P.), Queva1 (R.) & 36,07 \\
\hline Bos indious & $\begin{array}{l}\text { (2) zébu } \\
\text { arabe }\end{array}$ & 108 & 70 & 38 & Petit (J.P.), Queva1 (R.) & 35,19 \\
\hline $\begin{array}{l}\text { Groupe zébu } \\
\text { indien selon } \\
\text { Joshi } \\
\end{array}$ & $\begin{array}{l}\text { (3) Kankrej } \\
\text { (4) Malvi }\end{array}$ & $\begin{array}{l}230 \\
200\end{array}$ & $\begin{array}{l}115 \\
141\end{array}$ & $\begin{array}{r}115 \\
59\end{array}$ & Naik (S.N.), Baxi (J.) & $\begin{array}{l}50,00 \\
29,50\end{array}$ \\
\hline II & (5) Rath & 126 & 86 & 40 & Naik (S.N.), Baxi (J.) & 31,75 \\
\hline III & $\begin{array}{l}\text { (6) Dangi } \\
\text { (7) Gir }\end{array}$ & $\begin{array}{l}220 \\
200\end{array}$ & $\begin{array}{l}131 \\
138\end{array}$ & $\begin{array}{l}89 \\
62\end{array}$ & $\begin{array}{l}\text { Naik }(\mathrm{S.N.}), \operatorname{Baxi}\left(\mathrm{J}_{+}\right) \\
\text {Naik }\left(\mathrm{S} . \mathrm{N}_{+}\right), \operatorname{Baxi}(\mathrm{J})\end{array}$ & $\begin{array}{l}40,45 \\
31,00\end{array}$ \\
\hline IV & (8) Khillari & 210 & 135 & 75 & Naik (S.N.), $\operatorname{Baxi}$ (J.) & 35,71 \\
\hline
\end{tabular}

TABLEAU $\mathrm{N}^{\circ} \mathrm{II}$

Tests globaux indiquant le rôle très particulier de la race Kankrej qui masque toutes les autres différences.

\begin{tabular}{|c|c|c|c|c|c|}
\hline $\begin{array}{c}\text { Races comparées } \\
\text { du point de vue } \\
\text { déficience en G-6-PD }\end{array}$ & $\begin{array}{c}\text { Valeur } \\
x^{2}\end{array}$ & $\begin{array}{l}\text { Nombre } \\
d \cdot d \cdot 1 .\end{array}$ & $\mathrm{S}$ & N.S. & $\begin{array}{c}\text { Seuil de } \\
\text { signification }\end{array}$ \\
\hline $\begin{array}{l}\text { Race Kankrej (3) inclue } \\
\begin{array}{rrrrrr}12 & 34 & 5 & 6 & 7 & 8 \\
3 & 4 & 5 & 6 & 7 & 8 \\
3 & 4 & 5 & 6 & 8 \\
3 & 5 & 6 & 7 & 8 \\
3 & 5 & 6 & 8 \\
\text { Race Kankrej exclue } \\
1245 & 6 & 7 & 8 \\
4 & 5 & 6 & 7 & 8 \\
5 & 6 & 7\end{array}\end{array}$ & $\begin{array}{r}27,558 \\
27,322 \\
23,185 \\
21,032 \\
14,605 \\
7,387 \\
7,252 \\
4,871\end{array}$ & $\begin{array}{l}7 \\
6 \\
4 \\
4 \\
3\end{array}$ & $\begin{array}{l}x \\
x \\
x \\
x \\
x\end{array}$ & $\begin{array}{l}\mathrm{x} \\
\mathrm{x} \\
\mathrm{x}\end{array}$ & $\begin{array}{l}1 \mathrm{p} .1000 \\
1 \mathrm{p} .1 .000 \\
1 \mathrm{p} .1 .000 \\
1 \mathrm{p} .1 .000 \\
1 \mathrm{p} .100\end{array}$ \\
\hline
\end{tabular}

Les numéros indiqués dans la première colonne sont ceux correspondant aux diverses races selon ce qui est indiqué dans le tableau $I$. 
TABLEAU $\mathrm{N}^{\mathrm{b}}$ IIT

Comparaison de toutes les races êtudiées aux races Kankrej et Dangi.

\begin{tabular}{|c|c|c|c|c|c|c|}
\hline \multicolumn{3}{|c|}{ Races comparées } & $\begin{array}{l}\text { Valeur du } x^{2} \\
\text { calculée }\end{array}$ & $\begin{array}{c}\text { Seuil de } \\
\text { signification }\end{array}$ & Signification & \multirow[t]{2}{*}{$\begin{array}{l}\text { Valeur du } x^{2} \\
\text { selon Naik } \\
\text { et Collab. }\end{array}$} \\
\hline (3) $\mathrm{k}$ & Kankrej et & 1 & 3,75740 & & N.S. & \\
\hline & - & 2 & 6,51026 & 2 p. 100 & $\mathrm{~s}$ & \\
\hline & - & 4 & 18,66140 & $1 \mathrm{p} \cdot 1.000$ & $\mathrm{~s}$ & 21,3 \\
\hline & - & 5. & 11,03408 & $1 \mathrm{p} .1 .000$ & $\mathbf{S}$ & 11,04 \\
\hline & - & 6 & 4.13418 & $5 \mathrm{p} .100$ & $\mathrm{~S}$ & 4.10 \\
\hline & - & 7 & 15,94557 & $1 \mathrm{p} .1 .000$ & $\mathrm{~s}$ & 15,94 \\
\hline & - & 8 & 9.13082 & $1 \mathrm{p} .100$ & $\mathrm{~s}$ & 9,12 \\
\hline \multirow[t]{7}{*}{ (6) I } & Dangi et & 1 & 0,38496 & & N.S. & \\
\hline & - & 2 & 0,84769 & & N.S. & \\
\hline & - & 3 & 4,13418 & $5 \mathrm{p} \cdot 100$ & $\mathrm{~S}$ & 4,10 \\
\hline & - & 4 & 5,50884 & 2 p. 100 & $s$ & 5,56 \\
\hline & - & 5 & 2,59841 & & $\mathrm{~N} \cdot \mathrm{S}$. & \\
\hline & - & 7 & 4,06682 & $5 \mathrm{p} .100$ & $\mathbf{s}$ & 4,06 \\
\hline & - & 8 & 1,02326 & & N.S. & \\
\hline
\end{tabular}

Pour chaque ligne, on a 1 degrê de liberté (d.d,1.). On remarque surtout qu'on ne trouve aucune différence entre les zébus Kankrej et les taurins Kouri, 1'ancienneté des Kouri pouvant être rapprochée de celle qu'on reconnaît à la race Kankrej, sans qu'aucune conclusion puisse en être tirée.

Quand on compare les races deux à deux, aucune différence significative n'apparaît sauf quand la race Kankręj ou Dangi est concernée. Le tableau III expose les huit différences trouvées significatives dans les comparaisons où figurent ces deux races.

La race Kankrej semble avoir la particularité de posséder autant d'animaux déficients en G-6-PD que d'animaux normaux. C'est une race qui semble très ancienne, si l'on en juge par les dessins et les sculptures d'un sceau, trouvé dans les fouilles de MOHENJO-DARO au Pakistan (région du Sud). Ceux-ci représentent des types de bétail analogue, ils datent d'environ 3.000 avant J.C.

La race Malvi ressemblerait par certains aspects (du pelage et des cornes en particulier) à la race Kankrej, on ne note cependant aucune ressemblance du point de vue de la G-6-PD.

\section{DISCUSSION}

La première hypothèse de travail qui méritait d'être vérifiée concernait une éventuelle différence entre les deux grands types de bovins, taurins (Bos taurus) et zébus (Bos indicus). Les tests permettent d'abandonner cette hypothèse (tableau II, lignes 1 et 6 ) au moins jusqu'à ce qu'on puisse comparer un nombre plus important de données chez les diverses races de taurins. Il est tout de même assez curieux de constater que parmi toutes les races comparées au zébu Kankrej, seuls les taurins Kouri ne présentent pas de différence significative et de ce fait peuvent être considérés comme les plus proches quant à la déficience en G-6-PD.

La deuxième hypothèse concerne la classification des zébus de l'Inde et du Pakistan réalisée par JOSHI et PHILLIPS : elle trouverait un support biochimique intéressant si on pouvait établir une correspondance avec l'arsenal en G-6-PD dont chaque groupe dispose.

L'analyse des résultats de NAIK couplés aux nôtres, montre effectivement une différence significative entre les groupes suivants: I-I, I-III et I-IV; mais aucune entre les autres. $\mathrm{Si}$ on supprime la race Kankrej dans les comparaisons du groupe I, aucune ne demeure significative. L'ensemble des résultats permet donc de rejeter cette $2^{e}$ hypothèse et la race 
Dangi, qui semblait être différente des autres, ne l'est pas suffisamment pour caractériser le groupe III : les différences entre race sont donc plus importantes que celles entre groupe.

\section{CONCLUSION}

Il semble bien que la G-6-PD ne puisse pas être retenue comme base biochimique de la classification en groupe de JOSHI et PHILLIPS et qu'elle représente simplement une caractéristique raciale indépendante de la nature de zébu ou de taurin.

L'étude de la distribution de la déficience en G-6-PD chez les bovins amène à considérer deux groupes significativement différents.

Cette distribution a les caractéristiques suivantes : $m=36,21$ p. $100 \pm 6,75$ p. 100 . L'intervalle de confiance de la déficience moyenne en G-6-PD chez les bovins, calculée d'après cet échantillon de 8 races, se situe donc entre 42,96 p. 100 et 29,46 p. 100 . On voit tout de suite que seule la race Kankrej est à part dans cet échantillon et constitue à elle seule l'un des groupes.

Du point de vue de la déficience en G-6-PD, on peut donc constituer parmi les bovins étudiés deux groupes :

- groupe A: caractérisé par une déficience relativement grande en G-6-PD, située entre 40 et 50 p. 100 . On y range les races Kankrej et Dangi;

- groupe B : caractérisé par une déficience relativement faible en G-6-PD, située entre 36 et 25 p. 100 . Ce groupe comprend le zébu arabe, les autres races de zébus indiens, et, à la limite, les taurins Kouri.

Une étude plus importante des taurins devrait permettre de les ranger dans l'un des deux groupes, la place des Kouris pouvant rester intermédiaire.

\section{LISTE ALPHABETIQUE DES ABREVIATIONS}

- ARN : Acide ribonucléique.

- NAD : Nicotinamide-Adenine-dinucléotide. Nom moderne pour le DPN de Warburg. C'est un coferment de transhydrogénation sous sa forme oxydée (forme réduite: NADH). Il intervient dans la voie d'EmbdenMeyerhoff.

- NADP : C'est le phospho-NAD. Nom moderne pour le TPN de Warburg. C'est un coferment de transhydrogénation sous sa forme oxydée (forme réduite: NADPH). Il intervient dans le cycle des pentoses.

- Voie dEmbden-Meyerhoff : chaîne de dégradatión dụi glycogène par vore anaérobie, telle qu'elle se déroule dans le muscle.

\section{SUMMARY}

Biochemical polymorphism in cattle : glucose-6-phosphate dehydrogenase study

The authors recall the part of glucose-6-phosphate dehydrogenase in the pentose cycle in animals. Afterwards, they determine the erythrocyt enzym deficiency in 108 Arab zebu cattle and in 61 Kouri taurin cattle. The complete statistical analysis, including the data of NAIK and BAXI in 6 other breeds of indian and pakistan zebu, does not allow to hold as biological difference between Bos indicus and Bos taurus, the G-6-PD deficiency. It is not possible either to establish correlation with JOSHI'S and PHILLIPS' indian zebu cattle classification. Now one can think it is only a question of breed features in cattle.

\section{RESUMEN}

El polimorfismo bioquimico en los bovimos: Estudio de Ia glucosa-6-fosfato deshidrogenasa

Los autores indican el papel de la glucosa-6-fosfato deshidrogenasa en el ciclo de las pentosis en los animales. Despues, determinan la deficiencia de este enzimo eritrocitario en 108 cebus árabes y en 61 bovinos 
Kouri. El análisis estadístico completo de los resultados, volviendo a utilizar los datos integros de NAIK y BAXI sobre 6 otras razas de cebus de India y de Pakistan, no permite indicar como diferencia biologica entre cebus y bovinos la deficiencia de G-6-PD. No se puede tampoco establecer una correlación con la clasificación de los cebus de India de JOSHI y PHILIPS. Actualmente se puede pensar que solo se trata de una característica racial en los bovinos.

\section{BIBLIOGRAPHE}

1. BAXI (A. J.), NAIK (S. N.) et BHATIA (H. M.) Erythrocytic glucose-6-phosphate dehydrogenase in various animal species. Curr. Sci., 1963, 32: $405-406$.

2. BERGER (L.). The semi quantitative determination of the glucose-6-phosphate dehydrogenase activity in red cells or other fluids. Sigma Techn. Bull., 1965, 5 (400).

3. BOYER (S. H.), PORTER (I. H.) et WEILBA. CHER (R. G.). Electrophoretic heterogeneity of glucose-6-phosphate dehydrogenase and its relationship to enzyme deficiency in man. Proc. natn. Acad. Sci., U.S.A., 1962, 48: 1868.

4. CARSON (P. E.), FLANAGAN (C. L.), ICKES (C. E.), ALVING (A.S.). Enzimatic deficiency in primaquine-sensitive erythrocytes. Science, 1956, 124: 484.

5. JOSHI (N. R.) et PHILLIPS (R. W.). Les zébus de l'Inde et du Pakistan. Rome, F.A.O., 1955. (Coll. Etude agricole F.A.O. no 19.)

6. KHANOLKAR (V.R.), NAIK (S. N.), BAXI (A. J.) et BHATIA (H. M.). Studies in hemoglobin variants and glucose-6-phosphate dehydrogenase in Indian sheep and goat. Experimentia, 1963, 19: 472

7. KIRKMAN (H. N.) et HENDRICKSON (E. M.) Sex linked electrophoretic differences in glucose- 6-phosphate dehydrogenase. Am. J. hum. Genes., 1963, 15: 241 .

8. KORNBERG (A.). Enzimatic synthesis of Triphosphopyridine nucleotid. J. biol. Chem., 1950 182: 805 .

9. MARKS (P. A.) et BANKS (J.). Inhibition of mamalian glucose-6-phosphate dehydrogenase by steroïd. Proc. natn. Acad. Sci. U.S.A., 1960, 46 : 447.

10. MOTULSKY (A.G.) et CAMPBELL-KRAUT (J.M.). Proceeding of the Conference on genetic. Polymorphisms and geographic variations in disease N.Y. pp. 159-180.

11. NAIK (S. N.) et BAXI (A. J.), Glucose-6-phosphate dehydrogenase study in indian zebu cattle. XIth European Conference on Animal Blood Group and Biochemical polymorphism., 1968.

12. NAIK (S. N.), BAXI (A. J.) et BHATIA (H. M.). Blood groups, hemoglobin variants and glucose-6phosphate dehydrogenase study in the imported « jersey» cattle. Indian vet. J., 1963, 40 (11): 680.

13. WARBURG (O.) et CHRISTIAN (W.). Action d'un extrait soluble de globules rouges sur l'ester de Robinson. Biochem. Z., 1931, 238 : 131; 242 : 206. 